Article

\title{
Energy-Efficient Train Driving Strategy with Considering the Steep Downhill Segment
}

\author{
Wentao Liu ${ }^{1,2}$, Tao Tang ${ }^{1,2}$, Shuai Su ${ }^{1,3, *}$, Jiateng Yin ${ }^{1}$, Yuan Cao ${ }^{2,3}$ and Cheng Wang ${ }^{4}$ \\ 1 State Key Laboratory of Rail Traffic Control and Safety, Beijing Jiaotong University, Beijing 100044, China; \\ 17120250@bjtu.edu.cn (W.L.); ttang@bjtu.edu.cn (T.T.); jtyin@bjtu.edu.cn (J.Y.) \\ 2 School of Electronic and Information Engineering, Beijing Jiaotong University, Beijing 100044, China; \\ ycao@bjtu.edu.cn \\ 3 National Engineering Research Center of Rail Transportation Operation and Control System, \\ Beijing Jiaotong University, Beijing 100044, China \\ 4 School of IoT Engineering, Jiangnan University, Wuxi 214122, China; wangc@jiangnan.edu.cn \\ * Correspondence: shuaisu@bjtu.edu.cn
}

Received: 13 January 2019; Accepted: 31 January 2019; Published: 3 February 2019

check for updates

\begin{abstract}
Implementation of energy-efficient train driving strategy is an effective method to save train traction energy consumption, which has attracted much attention from both researchers and practitioners in recent years. Reducing the unnecessary braking during the journey and increasing the coasting distance are efficient to save energy in urban rail transit systems. In the steep downhill segment, the train speed will continue to increase without applying traction due to the ramp force. A high initial speed before stepping into the steep downhill segment will bring partial braking to prevent trains from overspeeding. Optimization of the driving strategy of urban rail trains can avoid the partial braking such that the potential energy is efficiently used and the traction energy is reduced. This paper presents an energy-efficient driving strategy optimization model for the segment with the steep downhill slopes. A numerical method is proposed to calculate the corresponding energy-efficient driving strategy of trains. Specifically, the steep downhill segment in the line is identified firstly for a given line and the solution space with different scenarios is analyzed. With the given cruising speed, a primary driving strategy is obtained, based on which the local driving strategy in the steep slope segment is optimized by replacing the cruising regime with coasting regime. Then, the adaptive gradient descent method is adopted to solve the optimal cruising speed corresponding to the minimum traction energy consumption of the train. Some case studies were conducted and the effectiveness of the algorithm was verified by comparing the energy-saving performance with the classical energy-efficient driving strategy of "Maximum traction-Cruising-Coasting-Maximum braking".
\end{abstract}

Keywords: rail transit; train control; energy-efficient driving strategy; steep downhill segment; local optimization

\section{Introduction}

Owing to the advantages in safety, high capacity and efficiency, urban rail transit has rapidly developed worldwide in recent years. However, with the massive construction and short headway, the energy consumption of urban rail systems has increased dramatically. Consequently, how to reduce the total energy consumption has become an important and urgent concern for a sustainable development of rail transit systems. The traction energy consumption of trains accounts for about $53 \%$ of the total energy consumption in urban rail transit system [1]. Thus, the total energy consumption of the system can be effectively decreased if the train traction energy consumption is reduced, which would also contribute to the reduction of operational cost and carbon emission [2]. 
Implementation of energy-efficient train driving strategy in the automatic train operation (ATO) system contributes greatly to reducing the traction energy consumption. In recent years, many scholars have conducted a lot of research on the energy-efficient train control problem, which is mainly divided into continuous control models and discrete control models.

In 1980, Milroy [3] developed an approach to optimize train speed trajectory based on the continuous train control model, which established the theoretical foundation of the optimal train control problem. Afterwards, the problem with constant gradients was analyzed and the Pontryagin maximum principle was applied to obtain the optimal speed trajectory by Asnis [4]. Howlett $[5,6]$ formulated a finite dimensional constrained optimization model and used the maximum principle to solve the energy-efficient train driving regimes and the corresponding switching points. Considering varying gradients and speed limits, Khmelnitsky [7] built a continuous train driving model, in which the kinetic energy was considered as the state variable. Liu [8] proposed an analytic algorithm to solve the optimal switching points among different regimes by applying the Pontryagin maximum principle. Taking variable traction efficiency into consideration, $\mathrm{Su}[9,10]$ developed a numerical algorithm based on an energy consumption allocation method, in which the energy-efficient driving strategy among multi-stations was calculated by optimizing the multi-station running time distribution. Except for the analytical and numerical algorithms, many scholars also used intelligent algorithms based on modern heuristic search methods to study the energy-efficient driving strategy of trains. To reduce traction energy consumption by making full use of coasting, Chang and Sim [11] applied the genetic algorithm to optimize the position of the coast starting points in the coast control table. Ma [12] used real-coded genetic algorithm to automatically calculate the optimal coasting points in the energy-efficient driving strategy of subway trains. Jin [13] used the neural network technology and genetic algorithm to study the energy-efficient driving strategy of trains on the undulating track, which could adapt to different line conditions and meet the requirements of real-time optimization. Ke et al. $[14,15]$ presented a method of designing block-layout between successive stations, in which the Max-Min ant colony algorithm was used to optimize the train speed curve for a significant improvement in computational efficiency.

In practice, the control output of the diesel electric locomotives used for the heavy freight is discrete. Each different handle position corresponds to a fixed fuel supply rate and output power, thereby determining that the traction cannot change continuously. Therefore, many scholars have developed discrete train control models for this feature [16]. In the 1990s, Cheng and Howlett [17,18] studied the energy-efficient driving problem with a constant gradient and speed limit, and proved that the energy-efficient driving regimes of discrete control model trains included "maximum traction, coasting, and maximum braking". Then, Pudney and Howlett [19] took varying speed limits into consideration, and proved that the train must run at limits on the segments where the speed limits were lower than the expected cruising speed. Besides, Howlett and Cheng [20] considered the problem of continuously changing gradients. They solved critical equations of switching points in different operating regimes by using Lagrange Function and Kahn-Tucker Conditions to find an optimal type of driving strategy. Importantly, Howlett [21] proved that an arbitrary continuous energy-efficient operating sequence can be approximated by discrete "traction-coasting" pairs, establishing a connection between continuous control and discrete control models. In addition, considering non-constant gradients, curve and speed limits, Han [22] used genetic algorithms to optimize the driving strategy of the train ATO system. Ding [23] also designed a genetic algorithm to find the optimal solution of the energy-efficient train operation problem. In 2014, based on a real ATO system, Dominguez [24] introduced a Multi-Objective Particle Swarm Optimization algorithm to obtain the consumption/time Pareto front, which solved the optimization problem more efficiently than the previous algorithms.

It is concluded from the above studies that the classical energy-efficient driving strategy consists of maximum traction, cruising, coasting, and maximum braking as well as their corresponding switching points [2]. However, if there are steep slope segments in the route, the position of the switching points 
will be affected. For an individual steep uphill segment, Howlett [25] introduced an analytical method to obtain the optimal switching points. Furthermore, Albrecht [26-28] proved that the optimal driving strategy always existed and was unique via a perturbation analysis. Considering the regenerative braking in the train model, Ko $[29,30]$ adopted dynamic programming to optimize the train driving strategy with the confined state space and irregular lattice for trains running on the route containing a steep downhill. For a steep downhill segment, if entering the ramp at a relatively high speed, the train would probably brake on the segment to avoid exceeding the speed limit, which increases the global energy consumption. If the entering speed is relatively low, the trip time would be longer and the operational efficiency and service quality would consequently be reduced. Hence, to make full use of the potential energy of the steep downhill segment, this paper proposes an approach to solving the energy-efficient control problem for trains running in the steep downhill segment based on the classical energy-efficient driving strategy.

The main contributions of this paper are stated as follows:

1. Considering the route with a steep downhill, the solution space for a given cruising speed is analyzed to obtain the classical energy-efficient driving strategy for a given trip time.

2. A local optimization approach is developed to reduce the traction energy consumption for trains running in the steep downhill segment by applying the dichotomization algorithm.

3. A global optimization is achieved with the utilization of the adaptive gradient descent method to calculate the optimal cruising speed, which corresponds to the minimum traction energy consumption.

The remainder of the paper is organized as follows. Section 2 describes the optimized problem including the formulation of an energy-efficient train control model as well as the definition of the steep downhill. In Section 3, a numerical method is designed to calculate the optimal driving strategy for trains running in a steep downhill segment. In Section 4, simulations with actual data of Beijing Yizhuang line is presented to illustrate the effectiveness of the proposed approach, followed by the conclusion in Section 5.

\section{Problem Description}

\subsection{Assumptions}

To simplify the train model, we make the following assumptions:

- The train is considered as a mass point when running on the track, and its mass is fixed.

- The slope of the centroid of the train represents the gradient of the entire vehicle.

- The traction efficiency is assumed to be constant, and the mechanical energy is used as the traction energy during the trip.

- The regenerative braking is not considered in the train model, because we only consider optimizing the driving strategy of a single train in this work.

\subsection{Model Formulation}

The train driving strategy, which is intuitively reflected with a train running speed curve, is the combination of an operating control sequence and the corresponding switching points among different control regimes. With the given planned trip time, line conditions, vehicle performances, etc., a set of train driving strategies between two successive stations satisfy the operation constraints [31] (see Figure 1).

Although the above driving strategies can make the train arrive at the target station on a punctual basis, the accelerating distances and positions are not the same between different driving strategies, resulting in different traction energy consumption for the interstation. The purpose of energy-efficient driving is to find a driving strategy with minimum traction energy consumption among these feasible strategies. 


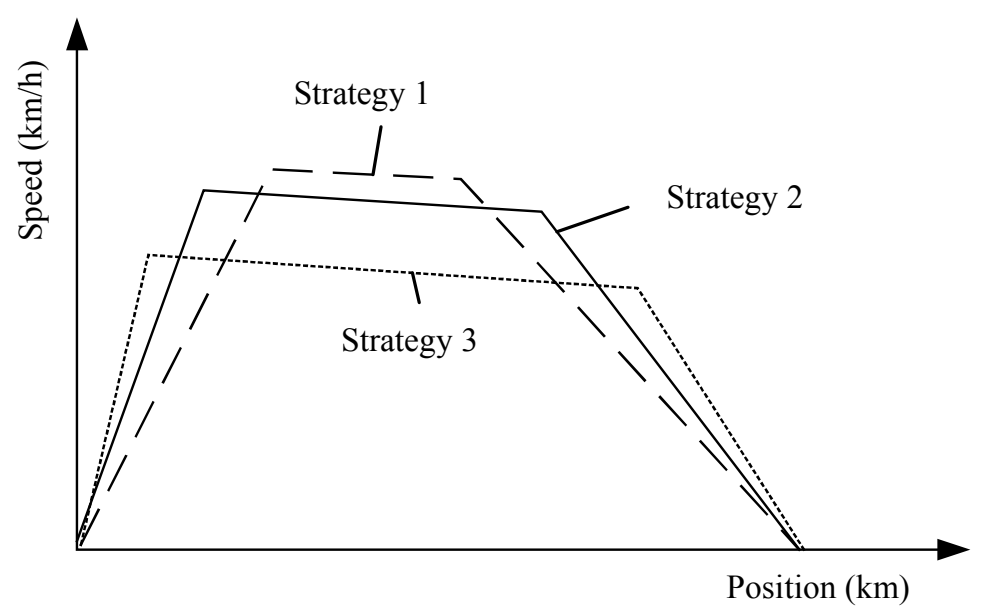

Figure 1. Different driving strategies of trains between successive stations.

The objective function of the energy-efficient train control problem can be generally written as [8]

$$
\min E=\int_{0}^{\mathcal{S}} u_{f} \cdot F(v) d x
$$

where $E$ represents the traction energy consumption; $u_{f}$ is the relative traction force; $F$ is the maximum traction force; $v$ is the train speed; and $x$ and $S$ are the train position and the trip distance, respectively.

For a mass-point train, the equation of motion can be described as [8]

$$
\left\{\begin{array}{l}
\frac{d v}{d t}=\frac{u_{f} F(v)-u_{b} B(v)-R_{b}(v)-R_{g}(x)-R_{c}(x)}{m \rho}, \\
\frac{d x}{d t}=v
\end{array}\right.
$$

where $m$ is the train mass; $\rho$ is the rotating mass factor; $u_{b}$ and $B$ denote the relative braking force and maximum braking force, respectively; $R_{b}(v)$ is the basic resistance including rolling resistance and air resistance; and $R_{g}(x)$ and $R_{c}(x)$ represent the grade resistance and curve resistance, respectively.

The traction force and the braking force should be bounded by the maximum traction and braking force. Thus, we have

$$
u_{f} \in[0,1], u_{b} \in[0,1] .
$$

The train speed should satisfy the maximum train speed and trip time constraints:

$$
0 \leq v(x) \leq V_{\max }(x) .
$$

where $V_{\max }$ is the maximum allowable train speed with respect to $x$.

To arrive at the next station on time and stop precisely, the boundary conditions of the train movement are described as follows.

$$
\begin{gathered}
v(0)=v_{\text {start }}=0, x(0)=x_{\text {start }}=0, \\
v(T)=v_{\text {end }}=0, x(T)=x_{\text {end }}=S .
\end{gathered}
$$

where $T$ is the planned trip time given by the timetable.

Based on Equations (1)-(5), the optimization model is formulated to minimize the energy consumption during the trip. 


\subsection{Definition of Steep Downhill}

The steep downhill is a piece of route where the train speed will increase without applying traction force (see Figure 2). In the steep downhill segment, the gradient force is larger than the sum of the curve resistance and line resistance.

$$
R_{g}(x)-\left(R_{c}(x)+R_{b}\left(v_{c}\right)\right)>0,
$$

In Equation (6), $R_{b}\left(v_{c}\right)$ can be described as follows [32]

$$
R_{b}\left(v_{c}\right)=m\left(a_{r} v_{c}^{2}+b_{r} v_{c}+c_{r}\right)
$$

$R_{g}(x)$ is calculated by

$$
R_{g}(x)=m g \sin \alpha(x),
$$

and the curve resistance can be described by empirical formulas [33]

$$
R_{c}(x)=f_{c}(r(x))=\left\{\begin{array}{l}
m \frac{6.3}{r(x)-55}, r(x) \geq 300 m \\
m \frac{4.91}{r(x)-30}, r(x)<300 m
\end{array},\right.
$$

where $a_{r}, b_{r}$, and $c_{r}$ are non-negative constants, which can be identified from the historical data. $g$, $\alpha(x)$ and $r(x)$ are the gravitational acceleration, the slope and the radius of curvature, respectively. With the estimate train speed for a fixed position, the basic and curve resistances can be calculated. By comparing with the gradient force, the steep downhill slope can be easily identified.

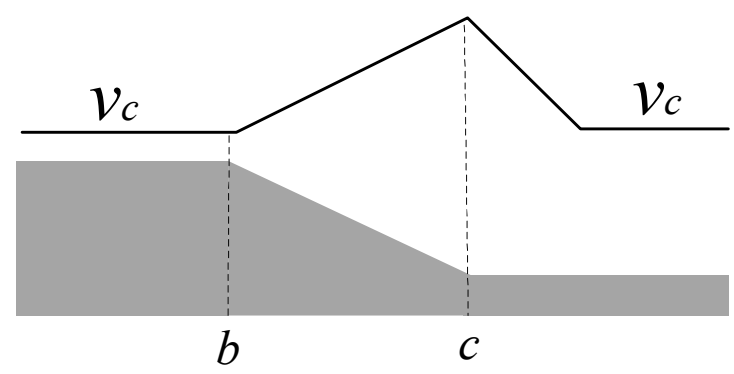

Figure 2. Train speed curve in a steep downhill segment.

\section{Solution Approach}

Given a specific planned trip time, the proposed solution algorithm starts from calculating the classical energy-efficient driving strategy with an initial cruising speed. With the obtained driving strategy, a local optimization approach is then developed to optimize the driving strategy in the steep downhill segment. Furthermore, an adaptive gradient method is applied to adjust the cruising speed such that the traction energy consumption during the trip is minimized.

The algorithm for solving the energy-efficient driving strategies of trains with considering the steep downhill segment is mainly divided into the following four parts and the overall framework of the proposed algorithm is shown in Figure 3.

- Initialization: Load line data and vehicle data and set a planned trip time $T$.

- Solution to classical energy-efficient driving strategy: Initialize a cruising speed $v_{\mathcal{c}}$, identify steep segment $[b, c]$ and solve classical energy-efficient driving strategy.

- Local optimization on $[b, c]$ : Judge the driving regime of the train in the steep downhill; if the train is cruising in this segment, then optimize the local driving strategy of the segment. 
- Optimal strategy search with adaptive gradient method: Calculate energy consumption and use the gradient method to solve the cruising speed corresponding to the minimum traction energy consumption, and then obtain the optimal energy-efficient driving strategy.

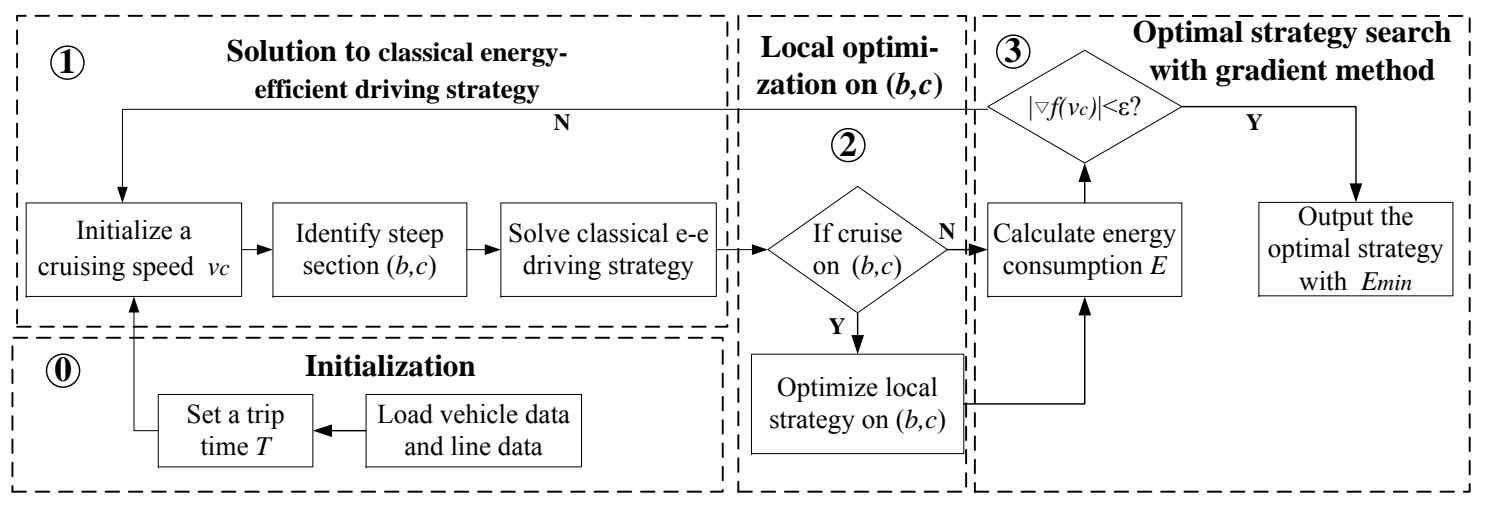

Figure 3. The structure of the proposed optimization approach.

\subsection{Calculation of Classical Energy-Efficient Driving Strategy}

\subsubsection{Analysis of Energy-Efficient Driving Regimes}

In this subsection, the energy-efficient driving regimes are analyzed by applying the Pontryagin maximum principle, according to which the following Hamiltonian function should be maximized with optimal control sequences $[8,10]$

$$
H=\frac{\eta_{1}(x)}{m \rho v} \times\left(u_{f} F-u_{b} B-R_{b}-R_{g}-R_{c}\right)+\eta_{2}(x) v-u_{f} F .
$$

where $\eta_{1}$ and $\eta_{2}$ and the complementary slackness condition $M(x)$ should satisfy the following differential equations:

$$
\begin{aligned}
& \frac{d v}{d t}=\frac{d H}{d \eta_{1}}, \quad \frac{d x}{d t}=\frac{d H}{d \eta_{2}}, \\
& \frac{d M}{d x}\left(v-V_{\text {max }}\right)=\frac{d H}{d \eta_{1}}, \quad \frac{d M}{d x} \geq 0
\end{aligned}
$$

Equation (10) can be rewritten as

$$
H=\left(\frac{\eta_{1}(x)}{m \rho v}-1\right) u_{f} F-\frac{\eta_{1}(x)}{m \rho v}\left(u_{b} B+R_{b}+R_{g}+R_{c}\right)+\eta_{2}(x) v .
$$

Thus, the four energy-efficient driving regimes are derived by maximizing Equation (12) in the following cases with respect to the control variables $u_{f}$ and $u_{b}$ [10]:

- $\quad \eta_{1}>m \rho v: u_{f}$ should be 1 and $u_{b}$ is 0 , which implies the Maximum traction regime.

- $\quad \eta_{1}=m \rho v: u_{b}$ should be 0 and $u_{f}$ may vary in $(0,1)$, which indicates the Partial traction phase;

$\eta_{1}=0: u_{f}$ should be 0 , and $u_{b}$ may vary in $(0,1)$, which suggests the Partial braking phase.

These two phases only exist for the Cruising regime by analyzing Equation (11).

- $\quad 0<\eta_{1}<m \rho v: u_{b}$ should be 1 and $u_{f}$ is 0 , which implies the Maximum braking regime.

- $\quad \eta_{1}<0$ : Both $u_{f}$ and $u_{b}$ should be 0 , which suggests the Coasting regime. 


\subsubsection{Solution Space Analysis}

For an individual given cruising speed, the steep downhill segment $[b, c]$ in the line is identified by Equation (6). Then, four critical states of the driving strategy, as shown in Figure 4, and the corresponding running time $t_{1}, t_{2}, t_{3}$ and $t_{4}$ are calculated. $t_{i}$ is explained as follows:

- $\quad t_{1}$ denotes the trip time of the driving strategy that the train begins to coast as soon as it reaches the cruising speed at the position $s_{1}$.

- $\quad t_{2}$ denotes the trip time of the driving strategy that the train begins to coast at the initial position $s_{2}$ of the steep slope.

- $\quad t_{3}$ denotes the trip time of the driving strategy that the train begins to coast at the final position $s_{3}$ of the steep slope.

- $t_{4}$ denotes the trip time of the driving strategy that the train begins to coast at the position $s_{4}$ when the train speed reaches the braking profile.
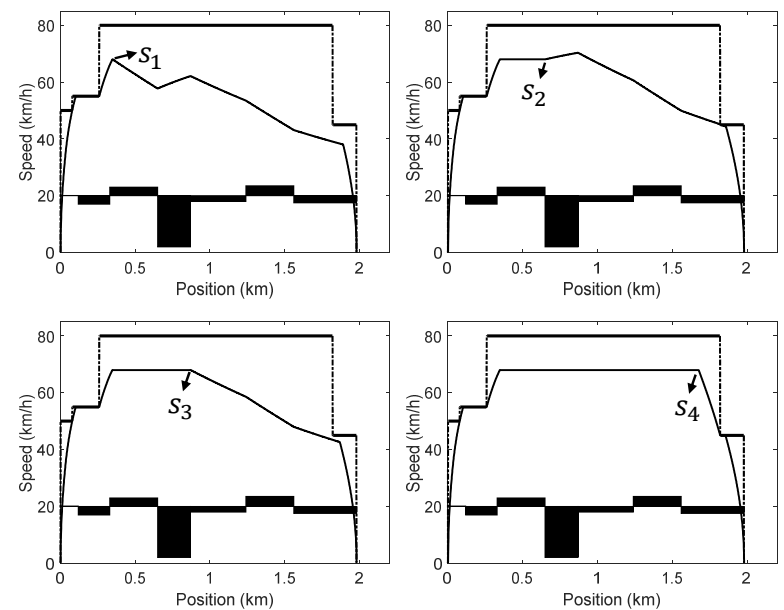

Figure 4. Driving strategies for the train, respectively, coasting from $s_{1}, s_{2}$, and $s_{3}, s_{4}$.

By the analysis, the length and position of the steep slope differ in the different steep slope lines, which leads to five typical scenarios of the running time $t_{1}, t_{2}, t_{3}$ and $t_{4}$ (see Figure 5).

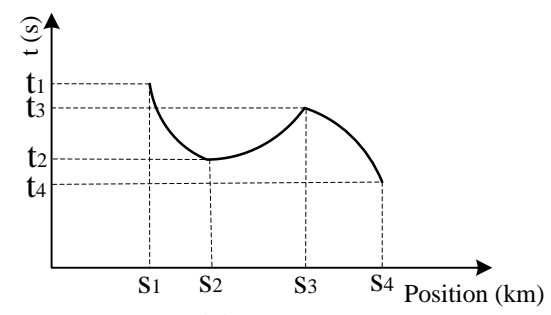

(a)

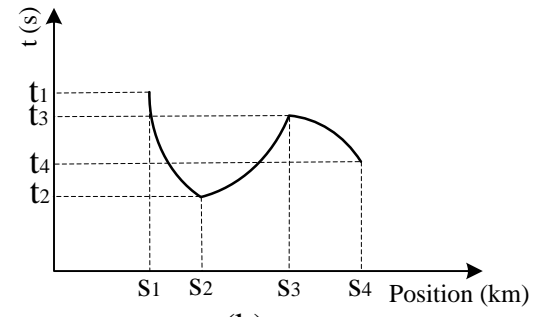

(b)

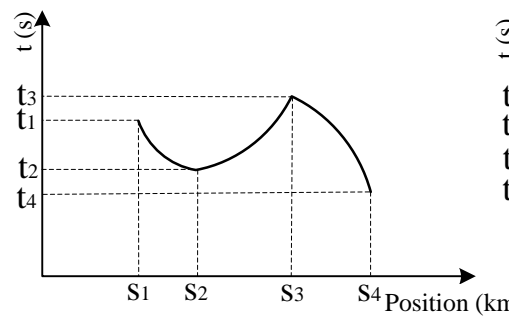

(c)

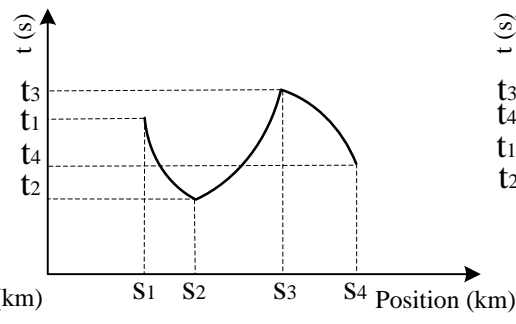

(d)

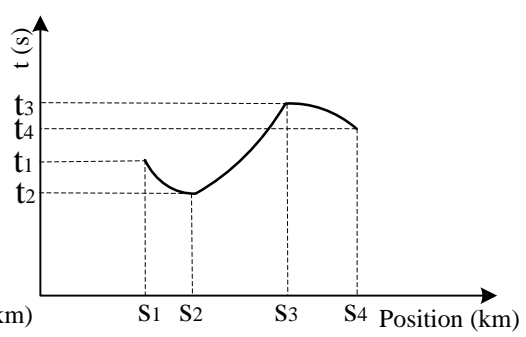

(e)

Figure 5. Five typical scenarios of the running time $t_{1}, t_{2}, t_{3}$ and $t_{4}$. (a) $t_{1}>t_{3}>t_{2}>t_{4}$; (b) $t_{1}>t_{3}>$ $t_{4}>t_{2} ;(\mathbf{c}): t_{3}>t_{1}>t_{2}>t_{4} ;(\mathbf{d}) t_{3}>t_{1}>t_{4}>t_{2} ;(\mathbf{e}) t_{3}>t_{4}>t_{1}>t_{2}$. 
- Scenario 1: $t_{1}>t_{3}>t_{2}>t_{4}$ (Figure 5a). This situation always happens for the scenario that the steep downhill is relatively short and exists in the middle of the line. For the segment $\left[s_{1}, s_{2}\right]$, when the train begins to coast later, the trip time with a higher average coasting speed will be shorter, i.e., $t_{1}>t_{2}$. Coasting from the position $s_{2}$ makes the train speed increase due to the steep slope such that the speed in the steep slope and the following segment is higher, which indicates $t_{3}>t_{2}$. In addition, a small steep slope has a small effect on the average train speed. Keeping the cruising speed on the segment $\left[s_{1}, s_{3}\right]$ can achieve a short trip time, i.e., $t_{1}>t_{3}$. The train speed will decrease without traction and steep slopes after $s_{3}$. Hence, we have $t_{3}>t_{4}$. In conclusion, $t_{1}$, $t_{2}, t_{3}$ and $t_{4}$ will satisfy $t_{1}>t_{3}>t_{2}>t_{4}$.

- Scenario 2: $t_{1}>t_{3}>t_{4}>t_{2}$ (Figure $5 \mathrm{~b}$ ). This situation generally happens for the scenario that the steep downhill exists in the second half of the line. Differing from the first situation, due to the steep slope exists in the second half, coasting from the position $s_{2}$ makes the average train speed higher than the cruising speed in the segment $\left[s_{2}, s_{4}\right]$, i.e., $t_{4}>t_{2}$. As a result, $t_{1}, t_{2}, t_{3}$ and $t_{4}$ will satisfy $t_{1}>t_{3}>t_{4}>t_{2}$.

- Scenario 3: $t_{3}>t_{1}>t_{2}>t_{4}$ (Figure 5c). This situation always happens when the steep downhill is relatively short and exists in the first half of the line. After accelerating to the cruising speed, the train immediately enters the steep downhill segment, and the speed rises to exceed the cruising speed such that the average running speed of the train in the segment $\left[s_{1}, s_{3}\right]$ is greater than the cruising speed, i.e., $t_{3}>t_{1}$. Thus, $t_{1}, t_{2}, t_{3}$ and $t_{4}$ will satisfy $t_{3}>t_{1}>t_{2}>t_{4}$.

- Scenario 4: $t_{3}>t_{1}>t_{4}>t_{2}$ (Figure 5d). This situation exists when the steep downhill is relatively long and exists in the first half of the line. Because a longer steep slope has a greater effect on the average train speed, coasting from the position $s_{2}$ makes the average train speed higher than the cruising speed for the segment $\left[s_{2}, s_{4}\right]$, i.e., $t_{4}>t_{2}$. Therefore, $t_{1}, t_{2}, t_{3}$ and $t_{4}$ will satisfy $t_{3}>t_{1}>t_{4}>t_{2}$.

- Scenario 5: $t_{3}>t_{4}>t_{1}>t_{2}$ (Figure 5e). This situation happens when the relatively long steep downhill exists in the first half of the line, and there is a downward slope, but not a steep downhill, in the segment $\left[s_{1}, s_{2}\right]$. Compared with Scenario 4, a downward slope in the segment $\left[s_{1}, s_{2}\right]$ brings a higher initial speed before stepping into the steep downhill, hence, the average train speed will be higher than the cruising speed in the segment $\left[s_{1}, s_{4}\right]$, that is, the trip time will be shorter $\left(t_{4}>t_{1}\right)$. Consequently, $t_{1}, t_{2}, t_{3}$ and $t_{4}$ will satisfy $t_{3}>t_{4}>t_{1}>t_{2}$.

\subsubsection{Coasting Position Calculation}

As shown in Figure 5, the running time $t$ in each section is monotonously increasing or decreasing. According to the planned trip time $T$ and the relationships among $t_{1}, t_{2}, t_{3}, t_{4}$, the distribution of the coasting position is firstly determined in Table 1.

Table 1. Five scenarios of $t_{1}, t_{2}, t_{3}$, and $t_{4}$ and distribution of the coasting point $s$.

\begin{tabular}{cccccccccc}
\hline \multicolumn{2}{c}{$\boldsymbol{t}_{\mathbf{1}}>\boldsymbol{t}_{\mathbf{3}}>\boldsymbol{t}_{\mathbf{2}}>\boldsymbol{t}_{\mathbf{4}}$} & \multicolumn{2}{c}{$\boldsymbol{t}_{\mathbf{1}}>\boldsymbol{t}_{\mathbf{3}}>\boldsymbol{t}_{\mathbf{4}}>\boldsymbol{t}_{\mathbf{2}}$} & $\boldsymbol{t}_{\mathbf{3}}>\boldsymbol{t}_{\mathbf{1}}>\boldsymbol{t}_{\mathbf{2}}>\boldsymbol{t}_{\mathbf{4}}$ & $\boldsymbol{t}_{\mathbf{3}}>\boldsymbol{t}_{\mathbf{1}}>\boldsymbol{t}_{\mathbf{4}}>\boldsymbol{t}_{\mathbf{2}}$ & $\boldsymbol{t}_{\mathbf{3}}>\boldsymbol{t}_{\mathbf{4}}>\boldsymbol{t}_{\mathbf{1}}>\boldsymbol{t}_{\mathbf{2}}$ \\
\hline$t_{2}>T>t_{4}$ & {$\left[s_{3}, s_{4}\right]$} & $t_{4}>T>t_{2}$ & {$\left[s_{1}, s_{2}\right]$} & $t_{2}>T>t_{4}$ & {$\left[s_{3}, s_{4}\right]$} & $t_{4}>T>t_{2}$ & {$\left[s_{1}, s_{2}\right]$} & $t_{1}>T>t_{2}$ & {$\left[s_{1}, s_{2}\right]$} \\
$t_{3}>T>t_{2}$ & {$\left[s_{1}, s_{2}\right]$} & $t_{3}>T>t_{4}$ & {$\left[s_{1}, s_{2}\right]$} & $t_{1}>T>t_{2}$ & {$\left[s_{1}, s_{2}\right]$} & $t_{1}>T>t_{4}$ & {$\left[s_{1}, s_{2}\right]$} & $t_{4}>T>t_{1}$ & - \\
$t_{1}>T>t_{3}$ & {$\left[s_{1}, s_{2}\right]$} & $t_{1}>T>t_{3}$ & {$\left[s_{1}, s_{2}\right]$} & $t_{3}>T>t_{1}$ & - & $t_{3}>T>t_{1}$ & - & $t_{3}>T>t_{4}$ & - \\
\hline
\end{tabular}

Taking the first scenario as an example, the detailed analysis process is described as the following:

- $\quad t_{2}>T>t_{4}$ or $t_{1}>T>t_{3}$ : In Figure $5 a$, the horizontal line from the ordinate $T$ intersects the curve in the figure at a unique point $s \in\left[s_{3}, s_{4}\right]$ or $s \in\left[s_{1}, s_{2}\right]$, which indicates the optimal coasting position with the trip time $T$ exists in the segment $\left[s_{3}, s_{4}\right]$ or $\left[s_{1}, s_{2}\right]$, and the solution is unique in these two situations. 
- $\quad t_{3}>T>t_{2}$ : There will be three solutions, which belong to $\left[s_{1}, s_{2}\right],\left[s_{2}, s_{3}\right]$ and $\left[s_{3}, s_{4}\right]$, respectively. Due to the principle that, when the running times are the same, a longer coasting distance contributes to a smaller traction energy consumption, the optimal coasting position should be found in $\left[s_{1}, s_{2}\right]$.

It is noted that cases such as $t_{3}>T>t_{1}$ in Scenario 3 should be treated differently. As shown in Figure $5 c$, if the planned trip time $T$ satisfies $t_{3}>T>t_{1}$, there will be two different solutions that belong to $\left[s_{2}, s_{3}\right]$ and $\left[s_{3}, s_{4}\right]$, respectively. By applying the principle that a longer coasting distance leads to a smaller traction energy consumption, the solution belonging to $\left[s_{3}, s_{4}\right]$ is undesirable. Additionally, if the train switches from cruising to coasting in position $s_{c}$ that belongs to $\left[s_{2}, s_{3}\right]$, the potential energy in the first half of the segment will be wasted (see Figure 6). Compared to the strategy that trains begin to coast at the position $s$, the trip time is the same but the latter strategy will cost less energy consumption, i.e., $E_{s_{c}}>E_{s}$. Thus, the solution $s_{c}$ is also undesirable. In addition, though the strategy with coasting from $s$ is dropped as the speed of $s$ is less than the current given cruising speed, it will be found in another circumstance when the other cruising speed is given.

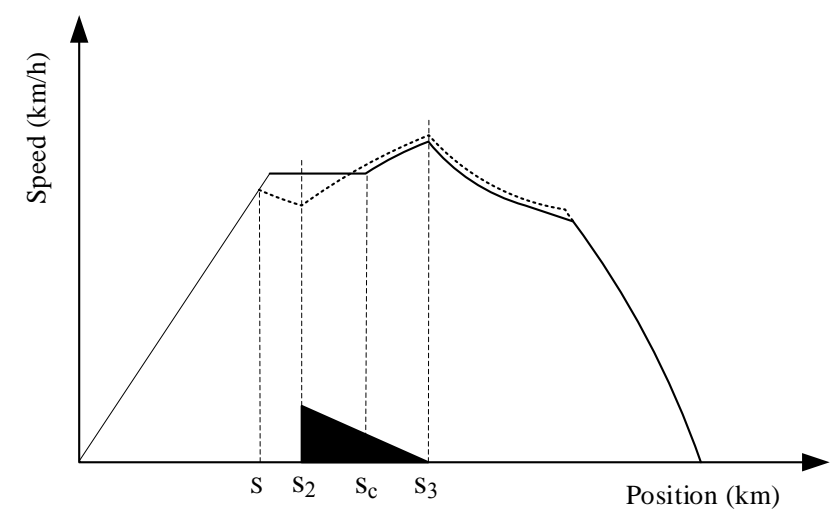

Figure 6. Driving strategies with coasting from $s$ and $s_{c}$, respectively.

Summarizing Sections 3.1.2 and 3.1.3, the starting point $s$ of the coasting regime is found according to the time of four critical states and the given trip time, and then a classical energy-efficient driving strategy with a fixed cruising speed is obtained.

\subsection{Local Optimization on Steep Downhill Segment}

As shown in Figure $7,[b, c]$ is the steep downhill segment. If the train uses the cruising regime at the segment $[a, d]$ in the classical energy-efficient driving strategy, the speed profile is shown as the horizontal dotted line and the running time in $[a, d]$ is $T_{\text {hold }}$. Specifically, the train may apply partial traction at the segment $[a, b]$ to maintain the cruising speed $v_{c}$. The traction energy consumption at such segment is $E_{(a, b)}$. The train applies partial braking at the segment $[b, c]$ to maintain the train drive at the cruising speed. The traction energy consumption at this segment is $E_{(b, c)}=0$. The regime at segment $[c, d]$ is the same as that at the segment $[a, b]$, and the traction energy consumption is $E_{(c, d)}$. Thus, before optimizing the driving strategy of steep downhill segment, the actual traction energy consumption at the segment $[a, d]$ can be expressed as

$$
E_{(a, d)}=E_{(a, b)}+E_{(c, d)} .
$$

Meeting the trip time constraint, the proposed local optimal driving strategy in the steep downhill is to switch the regime from cruising to coasting at point $x=a$ before the steep segment and from coasting to cruising at point $x=d$ after passing by the steep segment. The train coasts during the segment $[a, d]$ and the coasting time is $T_{\text {coast }}$. In this way, the potential energy of the steep downhill segment can be fully used. Furthermore, substituting the cruising with the coasting consumes no 
traction energy, i.e., $E_{(a, d)}^{\prime}=0$. Hence, the traction energy consumed by the optimized energy-efficient driving strategy is obviously reduced compared to the classical driving strategy [2], i.e., we have the following inequality:

$$
E_{(a, d)}>E_{(a, d)}^{\prime} .
$$

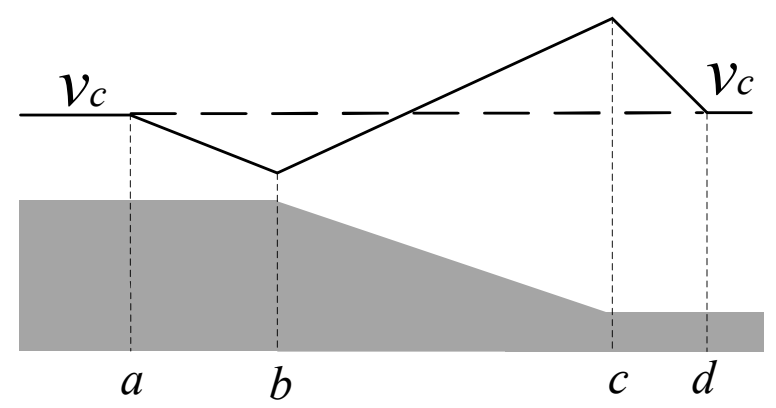

Figure 7. Local optimization at the steep downhill segment.

The trip time for the segment $[a, d]$ is continuous with respect to the coasting position $s$. The "s*" must exist, which satisfies

$$
T_{\text {hold }}=T_{\text {coast }} .
$$

Equations (14) and (15) indicate that the effect of local optimization in the steep slope segment is realized with the same trip time. To obtain the optimal coasting position $s^{*}$, the dichotomization method is used in this paper.

Specifically, we define a time function

$$
T\left(s_{a}\right)=T_{\text {hold }}\left(s_{a}\right)-T_{\text {coast }}\left(s_{a}\right),
$$

then the purpose of the dichotomization algorithm for calculating the coasting position is to find the position $s^{*}$ that satisfies

$$
s^{*}=\underset{s_{a}}{\arg }\left(T\left(s_{a}\right)=0\right) .
$$

The specific steps of the dichotomization algorithm can be described as Algorithm 1 .

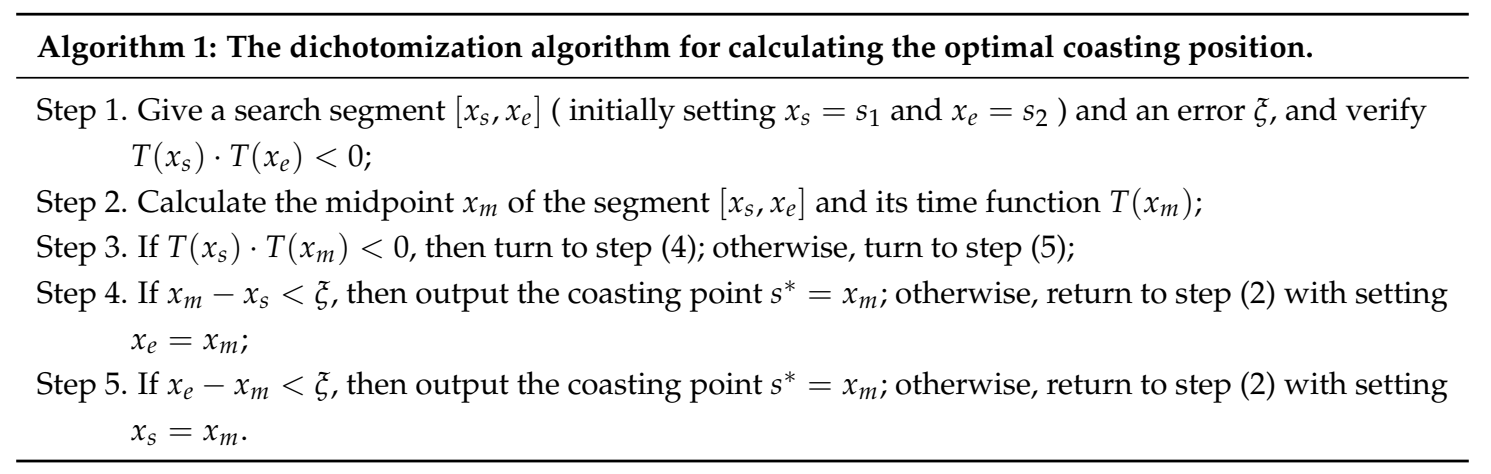

\subsection{Calculation of the Optimal Cruising Speed With Adaptive Gradient Descent Method}

The above subsections have described how to obtain an energy-efficient driving strategy for a fixed cruising speed. In this subsection, the adaptive gradient descent (AGD) method is applied to solve the optimal cruising speed corresponding to the minimum traction energy consumption of the trip. By dynamically incorporating knowledge of the geometry of the gradient in earlier iterations, AGD is able to perform informative gradient-based search, which ensures more robust performance [34]. 
The objective of gradient search is to find the optimal cruising speed

$$
v_{c}^{*}=\underset{v_{c}}{\arg \min } E\left(v_{c}^{k}\right) .
$$

The specific solution to the strategy search problem is described in Algorithm 2.

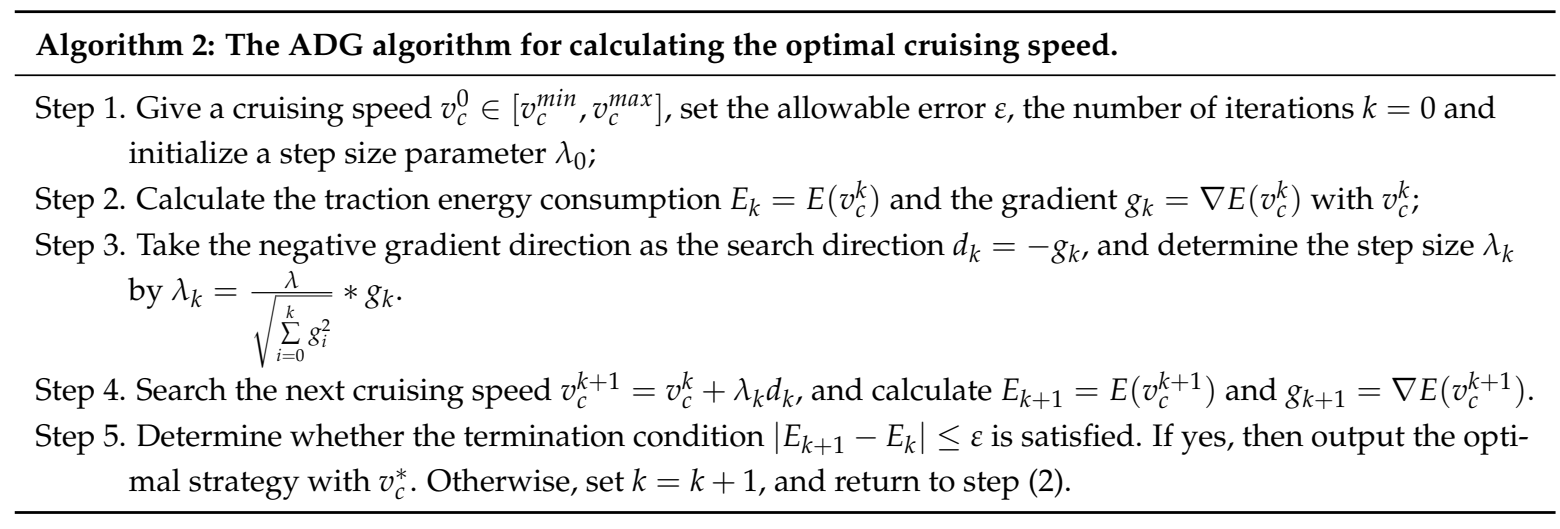

\section{Simulation Results}

In this Section, we present some simulations to illustrate the effectiveness of the proposed optimization approach. In Case 1, a case study based on the practical data of Yizhuang line was conducted and a comparison of energy efficiency was derived between the two algorithms, i.e., the proposed and the classical algorithms. In Case 2 , the route was gently modified to verify the availability of the proposed approach in the different situations shown in Figure 5.

\subsection{Case 1}

In this case, we chose the interval from Jinghai to Ciqu as an example. The distance between Jinghai and Ciqu stations is $2086 \mathrm{~m}$ and the speed limit of the whole segment is $80 \mathrm{~km} / \mathrm{h}$. The gradient information is shown in Table 2. The vehicle data of Beijing Yizhuang rail transit line were used for simulations. Characteristics of the traction force $F(v)$, the braking force $B(v)$ and the basic resistance $R_{b}(v)$ can be found in [10].

Table 2. Gradient information between Jinghai and Ciqu.

\begin{tabular}{cc}
\hline Segments $(\mathbf{m})$ & Gradients (\%) \\
\hline $0-19$ & -1.4006 \\
$20-339$ & 0 \\
$340-689$ & -15.6250 \\
$690-1389$ & -24.3900 \\
$1390-1629$ & 3.0030 \\
$1630-1979$ & -10.1010 \\
$1980-2086$ & -2 \\
\hline
\end{tabular}

For the cruising speed ranging $65-78 \mathrm{~km} / \mathrm{h}$, the segment from $690 \mathrm{~m}$ to $1389 \mathrm{~m}$ was identified as the steep downhill segment according to Equation (6).

When the planned trip time T was set to be $160 \mathrm{~s}$, the energy-efficient driving strategies calculated with the classical algorithm and the proposed approach are shown in Figure 8. As shown in Figure 8a, the cruising speed of the classical energy-efficient driving strategy is $69 \mathrm{~km} / \mathrm{h}$ and the traction energy consumption is $16.56 \mathrm{~kW} \cdot \mathrm{h}$. 
Additionally, Figure $8 \mathrm{~b}$ shows that the optimal cruising speed solved by the proposed approach is $72 \mathrm{~km} / \mathrm{h}$ and the traction energy consumption is reduced to $14.79 \mathrm{~kW} \cdot \mathrm{h}$. It is worth noting that there is no unnecessary braking on the steep slope segment in the optimized energy-efficient driving strategy, which consists of maximum traction regime in $\left[x_{1}^{\prime}, x_{2}^{\prime}\right]$, cruising regime in $\left[x_{2}^{\prime}, x_{3}^{\prime}\right]$, coasting regime in $\left[x_{3}^{\prime}, x_{4}^{\prime}\right]$ and maximum braking in $\left[x_{4}^{\prime}, x_{5}^{\prime}\right]$.

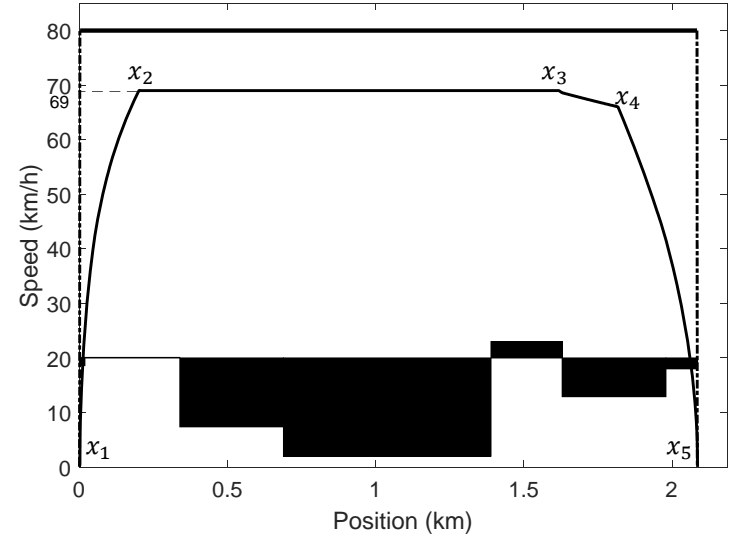

(a)

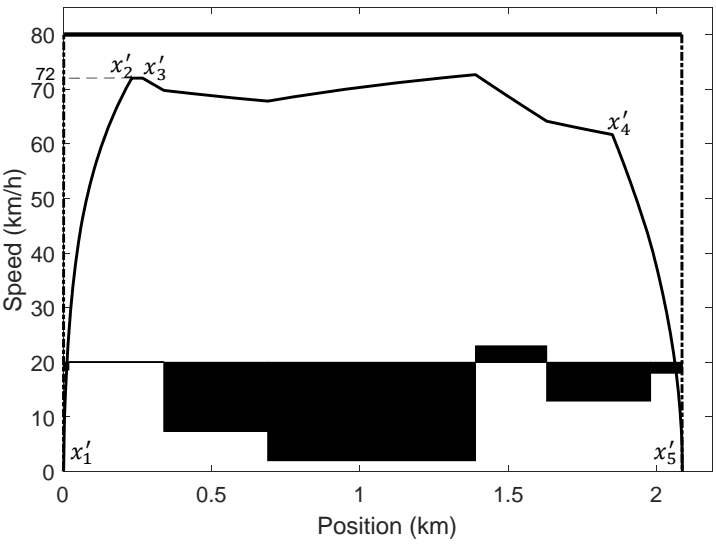

(b)

Figure 8. Energy-efficient driving strategies calculated with: (a) the classical algorithm; and (b) the proposed approach.

The energy-efficient driving strategies with the proposed approach and the classical energy-efficient algorithm were solved for other trip times. Then, the energy consumption was compared, as shown in Table 3.

Table 3. Energy efficiency comparison between the proposed and classical algorithm with different trip times.

\begin{tabular}{cccc}
\hline E (kW·h) & Methods & & \\
\cline { 1 - 3 } Trip Times & & & \\
\hline $155 \mathrm{~s}$ & 17.52 & 19.27 & $9.08 \%$ \\
$157 \mathrm{~s}$ & 16.35 & 18.48 & $11.52 \%$ \\
$160 \mathrm{~s}$ & 14.79 & 16.56 & $10.69 \%$ \\
$162 \mathrm{~s}$ & 13.84 & 15.38 & $10.01 \%$ \\
$164 \mathrm{~s}$ & 13.00 & 15.03 & $13.50 \%$ \\
Average & - & - & $10.96 \%$ \\
\hline
\end{tabular}

It is shown from the simulation results that the energy consumption is reduced by $10.96 \%$ in average. In addition, the energy-efficient performance is low when the trip time is short. To reduce the trip time, the train has to apply partial braking to keep a high speed, although the steep downhill segment exists. As a result, the potential energy cannot be used efficiently and the energy reduction is less in this situation.

The simulation was conducted on a personal desktop PC with processor speed of $3.6 \mathrm{GHz}$ and memory size of 16 GB. The average computation time of the proposed approach was about $0.12 \mathrm{~s}$, which can satisfy the requirement of real-time control. 


\subsection{Case 2}

As stated in Section 3.1, there are five kinds of lines, in which the length and position of the steep downhill segment are different. In this case, the availability of the proposed method was tested with the different line data (see Table 4).

Table 4. Gradient information of different lines.

\begin{tabular}{cccccc}
\hline Lines & Segments (m) & Gradients (\%) & Lines & Segments (m) & Gradients (\%o) \\
\hline & $0-120$ & 0 & $0-100$ & 0 \\
Line 1 & $121-330$ & -3 & $101-380$ & 3 \\
& $331-650$ & 3 & $381-660$ & -4 \\
& $651-870$ & -26 & Line 2 & $661-1200$ & 2.5 \\
& $871-1240$ & -2 & & $1201-1620$ & -28 \\
& $1241-1560$ & 3.5 & $1621-1880$ & 3.5 \\
& $1561-1980$ & -2.5 & $1881-1980$ & 2 \\
\hline \multirow{4}{*}{ Line 3 } & $0-100$ & 0 & $0-120$ & 0 \\
& $101-320$ & 3.5 & $121-420$ & 2 \\
& $321-460$ & 2 & $421-1400$ & -26 \\
& $461-780$ & -26 & Line 4 & $1401-1650$ & -2 \\
& $781-970$ & 3 & $1651-1800$ & \\
& $971-1320$ & -3 & $1801-2000$ & \\
& $1321-1580$ & 4 & $2001-2200$ & \\
& $1581-1760$ & -2 & & \\
$1761-1980$ & 2 & & \\
\hline
\end{tabular}

Taking Line 1 as an illustrative example, the planned trip time $T$ was set to be $160 \mathrm{~s}$. The optimal cruising speed calculated by the proposed approach is $75 \mathrm{~km} / \mathrm{h}$, and the corresponding energy consumption is $19.93 \mathrm{~kW} \cdot \mathrm{h}$. The energy-efficient driving strategies solved by the classical (the black line) and the proposed (the red line) approaches are shown in Figure 9. Obviously, th the proposed approach, the cruising regime is substituted with coasting on segment $\left[x_{3}, x_{4}\right]$ within the same trip time.

Under this circumstance, the critical states are $s_{1}=426 \mathrm{~m}, s_{2}=651 \mathrm{~m}, s_{3}=870 \mathrm{~m}$ and $s_{4}=1626 \mathrm{~m}$; the running times are $t_{1}=167.61 \mathrm{~s}, t_{2}=160.06 \mathrm{~s}, t_{3}=160.98 \mathrm{~s}$ and $t_{4}=151.91 \mathrm{~s}$; and the coasting position s, i.e., $x_{5}$ in Figure 9 , is $914 \mathrm{~m}$. It is clearly shown that, because $T$ is set to be within the time interval $\left(t_{4}, t_{2}\right]$, the coasting point $s$ is obtained from the interval $\left(s_{3}, s_{4}\right]$, which is in accordance with Scenario 1 described in Table 1, i.e., $s \in\left(s_{3}, s_{4}\right]$.

Furthermore, when different line conditions and planned trip times are given, the results solved by the proposed approach are shown in Tables 5 and 6 . Table 5 shows the optimal cruising speed and corresponding energy consumption of each scenario with the given planned trip time. Moreover, the critical states and the corresponding running time of each scenario are given in Table 6. It can be observed that the coasting point $s$ of each scenario is obtained according to the planned trip time, as analyzed in Section 3.1. For instance, the planned trip time $T$ of Line 2 was set as $160 \mathrm{~s}$, which belongs to the interval $(149.84,168.02]$, i.e., $T \in\left(t_{3}, t_{1}\right]$. Thus, according to Scenario 2 illustrated in Table 1 , the coasting point $s$ found in $(333,1201]$ is $622 \mathrm{~m}$, i.e., $s \in\left(s_{1}, s_{2}\right]$. 


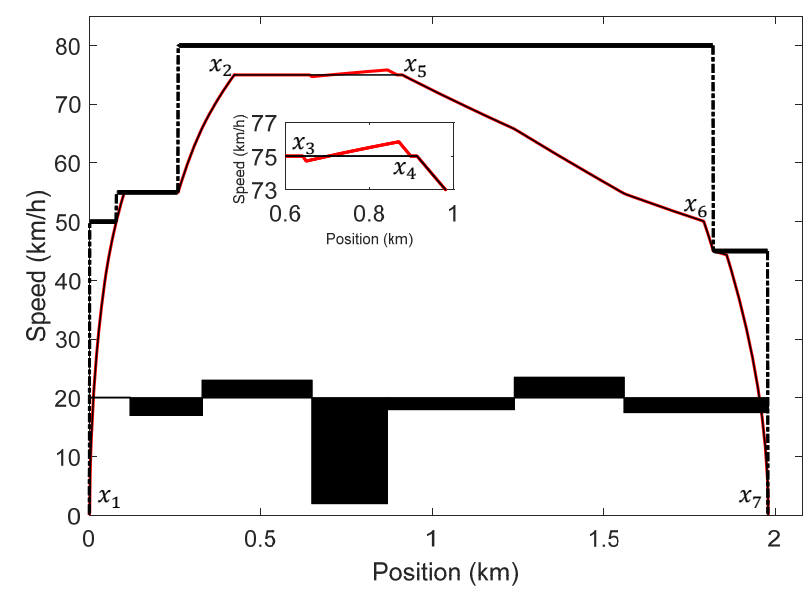

Figure 9. Energy-efficient driving strategy for the train running in Line 1 with $T=160 \mathrm{~s}$.

Table 5. The optimal cruising speed $v_{c}^{*}$ and energy consumption $E$ of each line with a planned trip time $T$.

\begin{tabular}{cccc}
\hline Lines & $\mathbf{T}(\mathbf{s})$ & $\boldsymbol{v}_{\boldsymbol{c}} \mathbf{( \mathbf { k m } / \mathbf { h } )}$ & $\mathbf{E} \mathbf{( k W \cdot h )}$ \\
\hline Line1 & 160 & 75 & 19.93 \\
Line2 & 160 & 74 & 19.19 \\
Line3 & 160 & 73 & 19.38 \\
Line4 & 164 & 73 & 15.85 \\
Line5 & 154 & 70 & 14.29 \\
\hline
\end{tabular}

Table 6. Four critical states $s_{1}-s_{4}$, the corresponding running time $t_{1}-t_{4}$ and the coasting point $s$ of different lines.

\begin{tabular}{cccccc}
\hline Lines & $s_{1}(\mathrm{~m})$ & $s_{2}(\mathrm{~m})$ & $s_{3}(\mathrm{~m})$ & $s_{4}(\mathrm{~m})$ & $s(\mathrm{~m})$ \\
\hline Line1 & 426 & 651 & 870 & 1626 & 914 \\
Line2 & 333 & 1201 & 1620 & 1689 & 622 \\
Line3 & 430 & 461 & 780 & 1579 & 1056 \\
Line4 & 319 & 421 & 1400 & 1906 & 343 \\
Line5 & 287 & 421 & 1400 & 1723 & 310 \\
\hline Lines & $t_{1}(\mathrm{~s})$ & $t_{2}(\mathrm{~s})$ & $t_{3}(\mathrm{~s})$ & $t_{4}(\mathrm{~s})$ & $T(\mathrm{~s})$ \\
\hline Line1 & 167.61 & 160.06 & 160.98 & 151.91 & 160 \\
Line2 & 168.02 & 149.22 & 149.84 & 149.76 & 160 \\
Line3 & 166.74 & 165.69 & 167.98 & 155.48 & 160 \\
Line4 & 164.56 & 162.21 & 166.26 & 161.77 & 164 \\
Line5 & 154.35 & 152.35 & 156.80 & 154.85 & 154 \\
\hline
\end{tabular}

In addition, we present comparisons among the proposed and the classical strategies to illustrate the energy-saving performance, respectively, with the same planned trip time (see Table 7). It can be concluded that the greater the proportion of the steep slope in the line accounts for, the more obvious the energy-saving effect is (e.g., see Line 4 and Line 5). 
Table 7. Energy-saving performance comparisons for trains running in different lines.

\begin{tabular}{lccc}
\hline E $\mathbf{( k W \cdot h )}$ & Methods & & \\
\hline Lines & & & \\
\hline & & & \\
& & & \\
Line 1 & 21.65 & 23.37 & $7.36 \%$ \\
Line 2 & 18.37 & 19.27 & $4.67 \%$ \\
Line 3 & 16.16 & 19.23 & $15.9 \%$ \\
Line 4 & 15.25 & 18.07 & $15.6 \%$ \\
Line 5 & - & - & $9.20 \%$ \\
Average & & & \\
\hline
\end{tabular}

\section{Conclusions}

Based on the classical energy-efficient train control approach, this paper proposes an optimization approach focusing on solving the energy-efficient control problem for trains running on a line with a steep downhill segment. The solution space for a given cruising speed is firstly analyzed to obtain the classical energy-efficient driving strategy for a given trip time. With the same trip time, a local optimization approach is developed to replace the partial braking in the downhill segment by the coasting regime such that the local energy consumption is reduced. Further, the adaptive gradient descent method is utilized to obtain the optimal cruising speed with minimum traction energy consumption to achieve a global optimization. Some simulations based on practical data of the Yizhuang line showed that the proposed approach can averagely reduce the energy consumption by $10.96 \%$, compared with the classical energy-efficient train control approach. Simulations with five typical lines were also conducted to indicate that the proposed method has a good availability for variable lines. The average computation time of this method was about $0.12 \mathrm{~s}$, thus the proposed approach can be applied in the real-time control system. Future work could extend this approach to study the energy-efficient train control strategy with considering multi-slopes in the line.

Author Contributions: Conceptualization, T.T.; Methodology, S.S., W.L.; Validation, J.Y.; Formal Analysis, Y.C., C.W.; Writing-Original Draft Preparation, W.L.; Writing-Review and Editing, S.S., W.L.; Funding Acquisition, S.S.

Funding: This research was funded by Beijing Laboratory of Urban Rail Transit, Beijing Key Laboratory of Urban Rail Transit Automation and Control, by the National Natural Science Foundation of China (U1734210, 61803021), the Beijing Natural Science Foundation "The Joint Rail Transit" (L171007) and the Fundamental Research Funds for the Central Universities (2018YJS195).

Conflicts of Interest: The authors declare no conflict of interest.

\section{References}

1. Su, S.; Tang, T.; Li, X.; Gao, Z.Y. Optimization on multi-train operation in subway system. IEEE Trans. Intell. Transp. 2014, 15, 673-684.

2. Liu, W.T.; Tang, T.; Su, S.; Chang, Y.H.; Li, X.L. Study on the energy-efficient driving strategy for trains running in the steep downhill section. In Proceedings of the International Conference on Electronic, Control Automation and Mechanical Engineering, Sanya, China, 19-20 November 2017; pp. 534-539.

3. Milroy, I.P. Aspects of Automatic Train Control. Ph.D. Thesis, Loughborough University, Loughborough, UK, 1980.

4. Asnis, I.; Dmitruk, A.; Osmolovskii, N. Control of the motion of a train by the maximum principle. Energy Optim. USSR Comput. Math. Math. Phys. 1985, 25, 37-44. [CrossRef]

5. Howlett, P.G. An optimal strategy for the control of a train. J. Aust. Math. Soc. B 1990, 31, 454-471. [CrossRef]

6. Howlett, P.G.; Milroy, I.P.; Pudney, P.J. Energy-efficient train control. Control Eng. Pract. 1994, 2, $193-200$. [CrossRef] 
7. Khmelnitsky, E. On an optimal control problem of train operation. IEEE Trans. Autom. Control 2000, 45, 1257-1266. [CrossRef]

8. Liu, R.F.; Golovitcher, I. Energy-efficient operation of rail vehicles. Transp. Res. A Policy Pract. 2003, 37, 917-932. [CrossRef]

9. Su, S.; Li, X.; Tang, T. Driving strategy optimization for trains in subway systems. Proc. Inst. Mech. Eng. F J. Rail Rapid Transit 2018, 232, 369-383. [CrossRef]

10. Su, S.; Tang, T.; Chen, L.; Liu, B. Energy-efficient train control in urban rail transit systems. Proc. Inst. Mech. Eng. F J. Rail Rapid Transit 2015, 229, 446-454. [CrossRef]

11. Chang, C.S.; Sim, S.S. Optimising train movements through coast control using genetic algorithms. IEE Proc. Electr. Power Appl. 1997, 144, 65-73. [CrossRef]

12. Ma, C.Y.; Mao, B.H.; Liang, X. Coast control of urban train movement for energy efficiency. J. Transp. Inf. Saf. 2010, 28, 37-42.

13. Jin, W.D.; Wang, Z.L.; Li, C.W. Study on optimal method of train operation for saving energy. J. China Railw. Soc. 1997, 6, 58-62.

14. Ke, B.R.; Chen, M.C.; Lin, C.L. Block-layout design using max-min ant system for saving energy on mass rapid transit systems. IEEE Trans. Intell. Transp. 2009, 10, 226-235.

15. Ke, B.R.; Lin, C.L.; Yang, C.C. Optimisation of train energy-efficient operation for mass rapid transit systems. IET Intell. Transp. Syst. 2012, 6, 58-66. [CrossRef]

16. Yang, X.; Li, X.; Ning, B. A survey on energy-efficient train operation for urban rail transit. IEEE Trans. Intell. Transp. 2016, 17, 2-13. [CrossRef]

17. Cheng, J.; Howlett, P.G. Application of critical velocities to the minimisation of fuel consumption in the control of trains. Automatica 1992, 28, 165-169.

18. Cheng, J.; Howlett, P.G. A note on the calculation of optimal strategies for the minimization of fuel consumption in the control of trains. IEEE Trans. Autom. Control 1993, 38, 1730-1734. [CrossRef]

19. Pudney, P.J.; Howlett, P.G. Optimal driving strategy for a train journey with speed limits. J. Aust. Math. Soc. B 1994, 1, 38-49. [CrossRef]

20. Howlett, P.G.; Cheng, J. Optimal driving strategies for a train on a track with continuously varying gradient. J. Aust. Math. Soc. B 1995, 3, 388-410. [CrossRef]

21. Howlett, P.G.; Milroy, I.P.; Pudney, P.J. Energy-efficient train control. In Advances in Industrial Control; Springer: Berlin, Germany, 1995.

22. Han, S. An optimal automatic train operation (ATO) control using genetic algorithms (GA). In Proceedings of the IEEE Region 10 Conference, Cheju Island, Korea, 15-17 September 1999; pp. 360-362.

23. Ding, Y.; Liu, H.; Bai, Y. A two-level optimization model and algorithm for energy-efficient urban train operation. J. Transp. Syst. Eng. Inf. Technol. 2011, 11, 96-101. [CrossRef]

24. Domínguez, M.; Fernández, A.; Cucala, A.P. Multi-objective particle swarm optimization algorithm for the design of efficient ATO speed profiles in metro lines. Eng. Appl. Artif. Intell. 2014, 29, 43-53. [CrossRef]

25. Howlett, P.G.; Pudney, P.J.; Vu, X. Local energy minimization in optimal train control. Automatica 2009, 45, 2692-2698. [CrossRef]

26. Albrecht, A.R.; Howlett, P.G.; Pudney, P.J.; Vu, X. Energy-efficient train control: From local convexity to global optimization and uniqueness. Automatica 2013, 49, 3072-3078. [CrossRef]

27. Albrecht, A.; Howlett, P.G.; Pudney, P.J. The key principles of optimal train control-Part 1: Formulation of the model, strategies of optimal type, evolutionary lines, location of optimal switching points. Transp. Res. B Meth. 2016, 94, 482-508. [CrossRef]

28. Albrecht, A.; Howlett, P.G.; Pudney, P.J. The key principles of optimal train control-Part 2: Existence of an optimal strategy, the local energy minimization principle, uniqueness, computational techniques. Transp. Res. B Meth. 2016, 94, 509-538. [CrossRef]

29. Ko, H.; Koseki, T.; Miyatake, M. Application of dynamic programming to the optimization of the running profile of a train. In Computers in Railways IX; WIT Press: Southampton, UK, 2004; pp. 103-112.

30. Ko, H.; Koseki, T.; Miyatake, M. Numerical study on dynamic programming applied to optimization of running profile of a train. IEEE Trans. Ind. Appl. 2005, 125, 1084-1092. [CrossRef]

31. Su, S.; Li, X.; Tang, T.; Gao, Z.Y. A subway train timetable optimization approach based on energy-efficient operation strategy. IEEE Trans. Intell. Transp. 2013, 14, 883-893. [CrossRef] 
32. Rochard, B.P.; Schmid, F. A review of methods to measure and calculate train resistances. Proc. Inst. Mech. Eng. F J. Rail Rapid Transit 2000, 214, 185-199. [CrossRef]

33. Wang, Y.H.; Schutter, B.D.; Ton, J.J. Optimal trajectory planning for trains-a pseudospectral method and a mixed integer linear programming approach. Transp. Res. C Emerg. Technol. 2013, 29, 97-114. [CrossRef]

34. John, D.; Elad, H.; Yoram, S. Adaptive subgradient methods for online learning and stochastic optimization. J. Mach. Learn. Res. 2011, 12, 2121-2159.

(C) 2019 by the authors. Licensee MDPI, Basel, Switzerland. This article is an open access article distributed under the terms and conditions of the Creative Commons Attribution (CC BY) license (http:/ / creativecommons.org/licenses/by/4.0/). 